\title{
Vitaminas C y E para la prevención de las complicaciones de la hipertensión en el embarazo. (1)
}

\author{
Roberts JM, Myatt L, Spong CY, Thom EA, Hauth JC, Leveno KJ, et al. N Engl J Med \\ 2010;362(14):1282-91. \\ Análisis crítico: Angie Vergara R. ${ }^{1}$, Jorge Carvajal C. $P h D^{2}$, Claudio Vera PG, MSc. ${ }^{2,3}$ \\ ${ }^{1}$ Posgrado de Obstetricia y Ginecología, ${ }^{2}$ Departamento de Obstetricia y Ginecología, Unidad de Medicina Materno \\ Fetal, 3 Unidad de Medicina Basada en Evidencias, Facultad de Medicina, Pontificia Universidad Católica de Chile.
}

\section{RESUMEN (1)}

Introducción: El estrés oxidativo ha sido propuesto como un mecanismo para relacionar una pobre perfusión placentaria, característica de la preeclampsia, con las manifestaciones clínicas de este desorden. Medimos los efectos de la suplementación con antioxidantes con vitaminas $\mathrm{C}$ y $\mathrm{E}$, iniciados precozmente en el embarazo, en el riesgo de eventos adversos severos, maternos, fetales y neonatales, relacionados con hipertensión asociada al embarazo. Métodos: Conducimos un estudio multicéntrico, randomizado, doble ciego, incluyendo mujeres nulíparas quienes tuvieran bajo riesgo de preeclampsia. Las mujeres fueron asignadas al azar a recibir suplementación diaria con $1000 \mathrm{mg}$ de vitamina $\mathrm{C}$ y $400 \mathrm{UI}$ de vitamina E, o placebo, entre la 9 y 16 semanas de embarazo. El evento de interés primario fue hipertensión severa asociada al embarazo o hipertensión leve o severa con aumento de enzimas hepáticas, trombocitopenia, aumento de los niveles de creatinina sérica, eclampsia, parto prematuro por indicación médica, restricción de crecimiento fetal, o muerte perinatal. Resultados: Un total de 10.154 mujeres fueron randomizadas. Los dos grupos fueron similares con respecto a las características basales y la adherencia a la droga del estudio. Resultados disponibles para 9.969 mujeres. No hubo diferencias entre los grupos de vitaminas y el placebo en la tasa del resultado primario $(6,1 \%$ y $5,7 \%)$, respectivamente; $R R$ grupo vitamina: 1,07 ; $95 \%$ IC: $0,91-1,25)$, o en la tasa de preeclampsia
(7,2\% y $6,7 \%$, respectivamente; RR: 1,$07 ; 95 \%$ IC: $0,93-1,24)$. La tasa de eventos adversos perinatales no difiere significativamente entre los grupos. Conclusiones: La suplementación con vitaminas $\mathrm{C}$ y $E$ iniciada en las semanas 9-16 del embarazo, en una corte no seleccionada de bajo riesgo, mujeres nulíparas, no reduce la tasa de eventos adversos maternos o perinatales relacionados a hipertensión asociada al embarazo.

\section{ANÁLISIS DE LA INVESTIGACIÓN}

\section{A. Relevancia clínica de la investigación}

La mayoría de las investigaciones se han centrado en población de alto riesgo de desarrollar preeclampsia, es interesante conocer el impacto de suplementación con vitaminas antioxidantes en población de bajo riesgo de síndrome hipertensivo del embarazo.

Escenario clínico: El estrés oxidativo podría relacionar la deficiencia en la perfusión placentaria que presentan las pacientes con preclampsia con los síntomas clínicos de la enfermedad. En los años 90, varios estudios en población de alto riesgo de desarrollar preclampsia, asociaron a la administración de antioxidantes con menor incidencia de la enfermedad. Sin embargo, revisiones sistemáticas de los estudios clínicos, tanto seguimiento de cohortes, como en estudios randomizados no han evidenciado beneficio del tratamiento con vitaminas antioxidantes (2-4). 


\section{B. EL ESTUDIO (1)}

Objetivo: Determinar si la suplementación de vitamina $C$ y $E$ en población de bajo riesgo de desarrollar preclampsia, previene las complicaciones asociadas a la hipertensión en el embarazo. Diseño: Estudio clínico randomizado, con ocultamiento de la secuencia, multicéntrico, estratificado por centros y ciego para pacientes e investigadores. $\mathrm{Pa}$ cientes: Se incluyó nulíparas, que no hubiesen tenido un embarazo mayor a 19 semanas, cursando embarazo único de 9 a 16 semanas. Se excluyeron pacientes con PAS $>135 \mathrm{mmHg}, \mathrm{PAD}>85 \mathrm{mmHg}$, proteinuria de 24 horas $>300 \mathrm{mg} / \mathrm{dl}$, antecedente de uso de hipotensores, uso de vitamina $C>150 \mathrm{mg}$ al día o vitamina $\mathrm{D}>75 \mathrm{UI}$. Pacientes con diabetes pregestacional, antecedentes de uso de AINES o de antiplaquetarios también fueron excluidas. Pacientes con antecedentes de metrorragia en el embarazo actual, malformación uterina. También se excluyeron pacientes con enfermedades concurrentes, aneuploidias fetales o malformaciones graves, embarazo por FIV y aquellas con antecedentes de abuso de drogas. Intervención: Suplementación con vitamina C (1000 mg) y vitamina E (400 UI) versus placebo. Resultado primario: Evento compuesto de síndrome hipertensivo del embarazo severo o bien moderado más uno de los siguientes: elevación de enzimas hepáticas (transaminasas o LDH), plaquetopenia, creatinina elevada, eclampsia, parto $<32$ semanas, restricción del crecimiento fetal, óbito $>20$ semanas, muerte neonatal. Como resultados secundarios: Preeclampsia moderada y otros eventos adversos maternos y fetales como muerte materna. Resultados: 31.917 pacientes eran posibles candidatas para participar en el estudio, de estas 13.113 fueron enroladas para evaluar adherencia al placebo; 2.959 pacientes se excluyeron, por no adherencia a tratamiento, rechazo voluntario al estudio, cumplieron algún criterio de exclusión, o no se presentaron al seguimiento; 10.154 pacientes fueron randomizadas; 5.088 al grupo de la intervención y 5.066 al grupo placebo. El seguimiento fue de $98 \%$. Como se observa en la Tabla I, no hubo diferencias significativas en el resultado primario medido, ni en ninguno de los resultados secundarios.

Tabla I

ANÁLISIS DE RIESGO

\begin{tabular}{|c|c|c|c|c|}
\hline Resultado & $\begin{array}{l}\text { Vitaminas } \\
(\%)\end{array}$ & $\begin{array}{l}\text { Placebo } \\
(\%)\end{array}$ & $\begin{array}{c}\mathrm{RR} \\
{[\mathrm{IC} 95 \%]}\end{array}$ & $\begin{array}{c}\text { RAR } \\
\text { [IC 95\%] }\end{array}$ \\
\hline Primario Compuesto & 6,1 & 5,7 & $\begin{array}{c}1,07 \\
{[0,91 \text { a } 1,25]}\end{array}$ & $\begin{array}{c}-0,004 \% \\
{[-0,013 \text { a } 0,005]}\end{array}$ \\
\hline Hipertensión severa & 4,2 & 4,1 & $\begin{array}{c}1,03 \\
{[0,85 \text { a } 1,24]}\end{array}$ & $\begin{array}{c}-0,001 \\
{[-0,009 \text { a } 0,007]}\end{array}$ \\
\hline \multicolumn{5}{|c|}{ Hipertensión moderada o severa más: } \\
\hline Enzimas hepáticas elevadas & 0,5 & 0,7 & $\begin{array}{c}0,79 \\
{[0,47 \text { a } 1,31]}\end{array}$ & $\begin{array}{c}0,002 \\
{[-0,001 \text { a } 0,005]}\end{array}$ \\
\hline Trombocitopenia & 0,4 & 0,6 & $\begin{array}{c}0,68 \\
{[0,39 \text { a } 1,17]}\end{array}$ & $\begin{array}{c}0,002 \\
{[-0,001 \text { a } 0,005]}\end{array}$ \\
\hline Creatinina $>1,5 \mathrm{mg} / \mathrm{dl}$ & 0,1 & 0,2 & $\begin{array}{c}0,63 \\
{[0,25 \text { a } 1,63]}\end{array}$ & $\begin{array}{c}0,001 \\
{[-0,001 \text { a } 0,003]}\end{array}$ \\
\hline Eclampsia & 0,2 & 0,1 & $\begin{array}{c}2,49 \\
{[0,78 \text { a } 7,94]}\end{array}$ & $\begin{array}{c}-0,001 \\
{[-0,003 \text { a } 0,001]}\end{array}$ \\
\hline Parto $<32$ sem & 0,3 & 0,3 & $\begin{array}{c}0,81 \\
{[0,39 \text { a } 1,68]}\end{array}$ & $\begin{array}{c}0,000 \\
{[-0,002 \text { a } 0,002]}\end{array}$ \\
\hline Restricción de crecimiento & 1,2 & 0,9 & $\begin{array}{c}1,30 \\
{[0,89 \text { a } 1,90]}\end{array}$ & $\begin{array}{c}-0,003 \\
{[-0,007 \text { a } 0,001]}\end{array}$ \\
\hline Muerte fetal o neonatal & 0,2 & 0,2 & $\begin{array}{c}1,09 \\
{[0,48 \text { a } 2,46]}\end{array}$ & $\begin{array}{c}0,000 \\
{[-0,002 \text { a } 0,002]}\end{array}$ \\
\hline
\end{tabular}

$\mathrm{RR}=$ Riesgo Relativo, IC = Intervalo de confianza, RAR= Reducción absoluta de riesgo. Calculos realizados con Catmaker ${ }^{\circledR}$, version 1,12004, diponible en www.cebm.net. 


\section{ANÁLISIS CRÍTICO}

Validez interna: Este estudio tiene muy bajo riesgo de sesgo, ya que las pacientes fueron asignadas aleatoriamente a los grupos, la asignación de pacientes a los distintos grupos fue oculta. Además durante el estudio fueron ciegos pacientes e investigadores. El seguimiento hasta el parto fue del $98 \%$ y los datos fueron analizados con intención de tratar. No hubo co-intervenciones y el estudio no fue detenido prematuramente. La tasa esperada del evento de interés compuesto se obtuvo de un estudio anterior "Prevención de la preclampsia en mujeres sanas nulíparas con bajas dosis de aspirina". Se calculó el tamaño muestral de 10.000 pacientes con un poder de $90 \%$ para mostrar una disminución del $30 \%$ en el evento de interés primario. Conclusión: El estudio tiene un diseño apropiado y no se detectaron fuentes de potencial sesgo. Así este estudio, de alta calidad metodológica, nos permite concluir que la suplementación con vitamina $C$ y $E$, iniciada entre las 9 y 16 semanas del embarazo, no reduce la frecuencia de hipertensión severa y/o complicaciones graves secundarias a la hipertensión en el embarazo, en pacientes de bajo riesgo de desarrollar preclampsia.

\section{BIBLIOGRAFÍA}

1. Roberts JM, Myatt L, Spong CY, Thom EA, Hauth JC, Leveno KJ, Pearson GD, Wapner RJ, Varner MW, Thorp JM Jr, Mercer BM, Peaceman AM, Ramin SM, Carpenter MW, Samuels P, Sciscione A, Harper M, Smith WJ, Saade G, Sorokin Y, Anderson GB; Eunice Kennedy Shriver National Institute of Child Health and Human Development Maternal-Fetal Medicine Units Network. Vitamins $C$ and $E$ to prevent complications of pregnancy-associated hypertension. N Engl J Med 2010;362:1282-91.

2. Polyzos NP, Mauri D, Tsappi M, et al. Combined vitamin $C$ and $E$ supplementation during pregnancy for preeclampsia prevention: a systematic review. Obstet Gynecol Surv 2007;62(3):202-6.

3. Spinnato JA 2nd, Freire S, Pinto E Silva JL, et al. Antioxidant therapy to prevent preeclampsia: a randomized controlled trial. Obstet Gynecol 2007;110(6):13118.

4. Rumbold AR, Crowther CA, Haslam RR, Dekker GA, Robinson JS; ACTS Study Group. Vitamins $C$ and E and the risks of preeclampsia and perinatal complications. N Engl J Med. 2006 Apr 27;354(17):1796-806. 